unmittelbaren Auswirkungen der Digitalen Revolution auf die Demokratie im 21. Jahrhundert. Formal finden sich einige kleinere Nachlässigkeiten bei der Edition, die den Lesefluss aber nicht stören. Die Aktualität des Themas, die breite Ausrichtung und die Tiefe der Beiträge macht den Tagungsband für eine große Zielgruppe lesenswert.

Christian Nestler

\title{
Extremismusforschung: aktuelle Einführung und langfristige Trends in gewohnter Qualität
}

Backes, Uwe, Alexander Gallus und Eckhard Jesse (Hrsg.): Jahrbuch Extremismus \& Demokratie (E \& D), 24. Jahrgang 2012, Nomos Verlagsgesellschaft, Baden-Baden 2012, 534 Seiten, $€ 54,-$

Hirscher, Gerhard und Eckhard Jesse (Hrsg.): Extremismus in Deutschland. Schwerpunkte, Vergleiche, Perspektiven (Reihe „Extremismus und Demokratie“, Band 26), Nomos Verlagsgesellschaft, Baden-Baden 2013, 608 Seiten, € 89,-.

In mittlerweile eingespielter Zusammenarbeit dreier Herausgeber entstanden, teilt sich der 24. Band des Jahrbuchs „E \& D“ in gewohnter Weise in die Abschnitte „Analysen“ (S. 13 - 101), „Daten, Dokumente, Dossiers“ (S. 105 - 261), „Literaturteil“ (S. 265 - 503) und Anhänge (S. 504 - 534). Bereits in den "Analysen“ kommt in exemplarischer Form die Problemzugangsweise der Herausgeber zum Ausdruck: Beiträge zu Demokratietheorie und Extremismus mit Äquidistanz nach links und rechts liefern einen Ordnungsrahmen, komplementäre Einzeluntersuchungen zu Detailaspekten von Linksterrorismus und historischem Rechtsextremismus vermitteln Einsichten in langfristige Trends, aktuelle Entwicklungen und zeitgeschichtliche Abläufe.

Extreme Fälle eines Wechsels aus der bürgerlichen Gesellschaft in den gewaltsamen Kampf gegen sie unter Abbruch einer hoffnungsvoll begonnenen akademischen Karriere zeichnet Alexander Gallus anhand von Akten aus der Studienstiftung des Deutschen Volkes über die ehemaligen Stipendiatinnen und Stipendiaten Ulrike Meinhoff, Horst Mahler, Gudrun Ensslin und Bernhard Vesper nach (S. 13 - 29). Die Fassungslosigkeit der Betreuer über Lebensweg und Ende der Geförderten werden vor allem durch Gallus 'Verzicht auf retrospektive Interpretationen plausibel nachvollziehbar.

Zeitgeschichtlichen Abläufen um die erfolglosen Rückkehrversuche des im Gegensatz zu seinem Bruder rechtzeitig aus Deutschland geflohenen „Alten Kämpfers“ der NSDAP Otto Straßer widmet sich Herbert Elzer (S. 72 - 101). Die auf Archivmaterialien gestützte Untersuchung fördert diverse Winkelzüge, Doppelbödigkeiten und Unaufrichtigkeiten in einem nach dem Zweiten Weltkrieg nicht mehr geschichtsmächtig gewordenen Zweig autoritären politischen Denkens zu Tage. Neben vergessenen finden sich teilweise auch prominente Namen, wobei der Autor aus Paul Freiherr von Schönaich-Carolath (S. 81) wenige Seiten später einen Prinzen macht (S. 88).

In übersichtlich-knapper Form ordnet und referiert Manfred G. Schmidt gekonnt Demokratietheorien unterschiedlicher Provenienz und mit divergierenden Prognosen (S. 30 
- 41). Lazaros Miliopoulos bietet in zeitgeschichtlicher Hinsicht ebenfalls einen Beitrag, dem es vor allem um die angemessene Strukturierung von Bekanntem geht. Allerdings entspricht die zeitliche Abgrenzung des Textes nicht ganz der Überschrift. Auch haben sich in die Darstellung einige Unschärfen und Fragwürdigkeiten eingeschlichen: Alois Hundhammer wird keineswegs eine altbayerisch-klerikal-konservativ geprägte Politik, sondern nur ein „föderalistische[r] Kurs“ zugeschrieben (S. 45), was für einen Landespolitiker nicht anstößig wäre. Im Gegensatz zu Elzers Darstellung soll Otto Straßer nicht aus Kanada, sondern aus den USA nach Deutschland zurückgekehrt sein (S. 49). Der BHE sei ebenso wie die DP komplett von CDU und CSU „aufgesogen“" worden (S. 50) - obwohl ein Vergleich zwischen Schleswig-Holstein und Hessen zeigt, dass sich nach Zurückdrängung des BHE je nach dessen vormaligem Koalitionspartner jahrzehntelang stabile Mehrheiten sowohl für die CDU als auch für die SPD haben ergeben können. CDU und CSU hätten sich in Gegenposition zur Ost- und „Bildungspolitik der Brandt-Regierung“ seit 1969 „verstärkt nach rechts orientiert“ (S. 55), was angesichts der föderativen Kompetenzverteilung auf dem Bildungssektor, der ambivalenten Haltung der CDU/CSU-Fraktion zur Ostpolitik und ihres hochgradig kooperativen Verhaltens zur Bundesgesetzgebung während der 6. Wahlperiode nachgerade höchst unplausibel erscheint. ${ }^{1}$

Gewohnt solide fallen dagegen die in diesem Jahrgang besonders breiten, aus verlässlichen Quellen zusammengetragenen Wahlanalysen Eckhard Jesses aus (S. 105 - 126). Deutlich tritt der quantitative und qualitative Unterschied zwischen dem gegenüber erklärten Systemgegnern selbst integrativ vorgehenden und seinerseits regional gesellschaftlich integrierten „weichen“ Linksextremismus der Linkspartei und dem schwerpunktmäßig in den gleichen Gegenden anzutreffenden, politisch-gesellschaftlich aber scharf ausgegrenzten „harten“ Rechtsextremismus der NPD hervor.

Uwe Backes liefert unter Auswertung amtlicher wie auch entlegener originärer Quellen vertiefte Einblicke in politische Entwicklungen sowie - zum Teil kriminelle - Betätigungen extremistischer Organisationen und ihres Umfeldes (S. 127 - 150), wobei ungeachtet der spektakulären NSU-Morde die registrierten Straftaten aus dem linksextremen Bereich in der Summe erneut höher ausgefallen sind als jene aus dem rechtsextremen Lager (2011: 1.157 zu 755, S. 136, S. 143). Eine Dokumentation zur „Pro“-Bewegung und analytische Artikel über extremistische Zusammenschlüsse innerhalb der Linkspartei (unter Bezeichnung Egon Krenz' als „letzten DDR-Staatsratsminister“, S. 172), zum Profil des verstorbenen Rechtsextremisten Jürgen Rieger, zur Integration von Muslimen in Deutschland, zur Extremismussituation in Schweden sowie über die leninistisch orientierte, aus der DKP heraus gegründete Zeitschrift „RotFuchs“ ergänzen das Bild. Besondere Aktualität kommt Armin Pfahl-Traughbers Aufarbeitung von Parallelen und Unterschieden zwischen den Aktivitäten der „NSU“-Gruppe und anderen rechtsextremistischen Gewalttaten zu (S. 180 196), eine Frage, der der Autor auch in der zweiten zu besprechenden Publikation nachgeht.

Virtuos-vielfältig ist erneut der breite Literaturteil angelegt, mit kenntnisreichen Auswertungen, kontroversen Stellungnahmen namhafter Autoren sowie Einzel- und Kurz-

1 Vgl. Jürgen Plöhn, 40 Jahre nach dem ersten Misstrauensantrag im Deutschen Bundestag: Probleme einer Minderheitsregierung am Beispiel der ersten Regierung Willy Brandts, in: ZParl, 44. Jg. (2013), H. 1, S. 76 - 92; Hans-Joachim Veen, Opposition im Bundestag, Bonn 1976. 
besprechungen. In ihrer Gesamtheit ergeben sie einen facettenreichen Überblick über das Themengebiet und dessen theoretische, zeithistorische und biographische Einbettung.

Der von Gerhard Hirscher und Eckhard Jesse herausgegebene, mit 608 Seiten ebenfalls stattliche Band „Extremismus in Deutschland“ ist von seiner Entstehung aus einer seit 2007 durchgeführten Veranstaltungsreihe der Hanns-Seidel-Stiftung geprägt. Hieraus resultiert ein stärkerer Bezug zu Themenaspekten mit besonderer Relevanz für die politische Bildung, der sich auch in einer inhaltsbezogenen, vermeintlich leicht nachvollziehbaren Grobgliederung niederschlägt. Abgehandelt werden „I. Rechtsextremismus“, „II. Linksextremismus“, „III. Islamismus“ und „IV. Vergleiche“. Unter den Autoren finden sich etablierte wie auch jüngere Fachleute. So trifft man in Einleitung und 29 Einzelbeiträgen bei Themen wie auch Autoren etliche „alte Bekannte“, allerdings zum Teil in neuer Besetzung und mit dem erfolgreichen Bemühen, auch grundsätzliche Themen durch aktuelle Aspekte neu zu akzentuieren.

Die jahrzehntelange Diskussion um das Konzept der „streitbaren Demokratie“ wird von Eckhard Jesse durch eine offensive Auseinandersetzung mit jüngsten Publikationen der Kritiker Claus Leggewie, Horst Meier und Wolfgang Wippermann fortgeführt (S. 505 - 526). Thematisch wird dieser aktuell-gehaltvolle Grundsatzartikel des „Altmeisters“ durch Beiträge der wissenschaftlichen Mitarbeiter Sebastian Liebold und Julia Gerlach zu Freiheitsbegriff (S. 349 - 369) und Vereinsverboten (S. 527 - 548) ergänzt. Neben Texten zum Selbstverständnis von Links- und Rechtsautonomen, zur Extremismusprävention, zum Verhältnis zwischen SPD und Linkspartei sowie einigen anderen stehen die genannten Texte im Abschnitt „Vergleiche“, was dem Begriff des Vergleichs weder in analytischer noch in deskriptiver Variante entsprechen dürfte.

Auch eine Studie zum „Wählerpotential von Rechts- und Linksaußenparteien“ (Matthias Dilling / Viola Neu, S. 397 - 434) ist diesem Abschnitt zugeordnet. Unter Verzicht auf den Extremismusbegriff halten darin Dilling und Neu für die Periode 1990 bis 2012 bei fünf Parlamentseinzügen von „Rechtsaußenparteien“ (einschließlich der „Republikaner“) in die zehn Parlamente der „alten“ und deren sieben in die fünf Landtage der „neuen“ Länder (jeweils ohne Berlin) die Wahlerfolge zwischen Ost und West für „relativ ausgeglichen“ (S. 404) - eine Sichtweise, die der Rezensent nicht zu teilen vermag. Als Interpretation für die Wanderung sächsischer PDS-Wähler zur NPD im Jahre 2004 wird allein eine „unideologische Protestwahl“ angeboten (S. 405). Aber entspräche es nicht Fraenkelschem Demokratie- und einem korrespondierenden Extremismusverständnis, bei Gegnern pluralistischer Demokratie als Element ihrer Persönlichkeitsstruktur eine Komponente autoritären Denkens für möglich zu halten, die links oder rechts ihren konkreten Ausdruck finden kann? ${ }^{2}$

International vergleichende Fragestellungen werden dagegen in den richtungsorientierten Abschnitten behandelt: Uwe Backes schreibt über qualitative und quantitative Dimensionen der Bedrohung durch rechtsextremistische Gewalt in Europa (S. 43 - 61), Tom Thieme über rechtsextremistische Parteien in den Staaten der EU seit 1990 (S. 27 - 42), Tom Mannewitz im Sinne eines asymmetrischen Vergleichs über Deutschlands parteiförmigen Linksextremismus in Europa (S. 133 - 151).

2 Bemerkungen zur politischen Anthropologie bei Ernst Fraenkel, Die Wissenschaft von der Politik und die Gesellschaft, in: ders. I Falk Esche / Frank Grube (Hrsg.), Reformismus und Pluralismus, Hamburg 1973, S. 337 - 353, besonders S. 340, S. 350, S. 352. 
Dabei präsentiert Thieme eine Tabelle zum Auftreten rechts- und linksextremistischer Parteien in Regierungen und Parlamenten, in der er den rechten Flügel des österreichischen Parteiensystems den „Antisystemparteien“ zurechnet (S. 30), was dessen weithin akzeptierter Charakterisierung als rechtspopulistisch ${ }^{3}$ widerspricht, ohne dass Thieme seine abweichende Auffassung diskutiert. Unplausibel erscheint es, wenn Bulgarien nur aufgrund der Partei „Ataka“ zu den Ländern mit Rechtsextremisten im Parlament gerechnet wird, die aus der kommunistischen Partei hervorgegangene BSP hingegen keine Berücksichtigung findet, wohingegen Thieme Deutschland angesichts seiner SED-Nachfolgepartei PDS beziehungsweise Linkspartei unter die Länder mit parlamentarisch aktiven Linksextremisten einordnet.

Gertrud Gleixner behandelt anhand der Anstrengungen des vormaligen NPD-Vorsitzenden Udo Voigt (bis November 2011) die Entwicklung der mit Abstand stärksten rechtsextremistischen Partei (S. 79 - 96). Deutlich zeichnet sich deren völkisch geprägte Systemgegnerschaft ab. Trotz seines letztlichen Scheiterns wird Voigt von Gleixner nach Effektivitätskriterien als wirkungsvoller Vorsitzender seiner Partei porträtiert, da er durch den Zusammenschluss mit der DVU einen organisatorischen, durch den Einzug der Partei in die Landtage von Sachsen und Mecklenburg-Vorpommern auch einen elektoralen Erfolg für sich in Anspruch nehmen konnte, auch wenn es ihm nicht gleang, die desaströse finanzielle Lage der NPD zu beheben und einen gesamtdeutschen Durchbruch zu erzielen.

Vor dem Hintergrund dieser Entwicklung der Partei liest man die Erörterungen Marc Brandstetters zu deren möglichem Verbot (S. 97 - 112). In Anknüpfung an die - sachlich angemessene - Haltung Eckhard Jesses zu dieser Frage dominieren auch bei Brandstetter die Contra-Argumente. Dabei hebt er das empirisch anhand verschiedener Aktivisten beobachtete Wechseln von Organisation zu Organisation und die im Falle eines etwaigen NPD-Verbots dafür schon bereitstehende Formation „Die Rechte“ hervor. Auch betont Brandstetter die eigenen Kriterien des Europäischen Gerichtshofes für Menschenrechte, vor dem ein Urteil des Bundesverfassungsgerichts Bestand haben sollte, was bei marginaler Größe und friedlichem Verhalten der NPD durchaus fraglich erscheine. Ungeachtet anerkennenswerter moralischer Gründe für einen Verbotsantrag wäre ein etwaiges Scheitern für hochmotivierte freiheitlich-demokratische Gegner der Partei ein demoralisierender Schlag.

Zum Islamismus diskutiert Helmut Albert die Gefährdungslage in Deutschland (S. 275 - 284), Herbert L. Müller die öffentliche Wahrnehmung von Gefährdungen (S. 305 - 326) und Michail Logvinov das Verhalten deutschstämmiger Salafisten zwischen Glaubensverbreitung und bewaffnetem Kampf (S. 285 - 304). Gestützt auf Kenner der arabischen Sprache analysiert Barbara Zehnpfennig die Ideologie des Islamisten Sayyid Qutb (S. 327 345), der die Führung von Al-Quaida beeinflusst hat. Zehnpfennig betont Parallelen zwischen Islamismus, Marxismus und Nationalsozialismus, verzichtet aber auf einen Vergleich islamistischer Lehren mit dem Christentum, obgleich dieses mit Paradies, Abfall, göttlichem Erlöser und endzeitlichem Frieden in relevanter Weise auch „häretische“ Strömungen wie Islam und Marxismus hat prägen können.

3 Vgl. Frank Decker (Hrsg.), Populismus in Europa, Wiesbaden und Bonn 2006; Everhard Holtmann / Adrienne Krappidel / Sebastian Rehse, Die Droge Populismus, Wiesbaden 2006; Florian Hartleb, After their establishment: Right-wing populist parties in Europe, Brüssel 2011; Fritz Plasser / Peter A. Ulram, Das Parteiensystem Österreichs, in: Oskar Niedermayer / Richard Stöss / Melanie Haas (Hrsg.), Die Parteiensysteme Westeuropas, Wiesbaden 2006, S. 351 - 372. 
Für jeden, der sich aktuell über Fragen des Extremismus in Deutschland und darüber hinaus informieren will, ist das neue Jahrbuch unverzichtbar. Der angezeigte Sammelband kann als aktuelle Einführung in die Extremismusproblematik gelesen werden. Eine systematisch überzeugendere Gliederung wäre allerdings möglich gewesen. Auch bleibt eine möglichst präzise Verwendung von Begriffen Wunsch des Rezensenten: Entgegen journalistischer Übung sollte nicht von „SPD-Bürgermeistern“, nicht von „Hochburgen“ nirgendwo gesellschaftlich tonangebender Kleinparteien und im Falle vorhandener parlamentarischer Stützfraktionen nicht von bloß „tolerierten“ Minderheitsregierungen die Rede sein. Aber diese weitgehend formalen Anmerkungen tun dem Wert der vermittelten Informationen keinen Abbruch.

Jürgen Plöhn

\section{Rechtsextremismus in Deutschland und Europa: wissenschaftliche Analysen und journalistische Beobachtungen}

Brandstetter, Marc: Die NPD unter Udo Voigt. Organisation, Ideologie, Strategie, Nomos Verlagsgesellschaft, Baden-Baden 2013, 402 Seiten, € 59,-.

Staud, Toralf und Johannes Radke: Neue Nazis. Jenseits der NPD: Populisten, Autonome Nationalisten und der Terror von rechts, Kiepenheuer \& Witsch, Köln 2012, 272 Seiten, € 9,99.

Langebach, Martin und Andreas Speit: Europas radikale Rechte. Bewegungen und Parteien auf Straßen und in Parlamenten, Orell Füssli-Verlag, Zürich 2013, 287 Seiten, € 21,95.

Bei der Kampfkandidatur um den Bundesvorsitz der „Nationaldemokratischen Partei Deutschlands“ (NPD) setzte sich beim Parteitag 2011 Holger Apfel gegen den bisherigen Amtsinhaber Udo Voigt durch. Damit ging für die rechtsextremistische Partei eine Ära zu Ende, nachdem sie zwischen 1996 und 2011 nach einem jahrzehntelangen Schattendasein erstmals wieder Erfolge bei Landtagswahlen hatte verbuchen können. Darüber hinaus entwickelte sich die NPD im rechtsextremistischen Lager zu einer dominierenden Kraft, die mit ihrem „Staubsauger“-Effekt die unterschiedlichsten Akteure anzog. Der Frage, wie in der Rückschau die „Ära Voigt“ der Partei eingeschätzt werden kann, widmet sich die umfangreiche Studie des Politologen Marc Brandstetter. Darin geht es um die Besonderheiten, die in der Entwicklung von der „alten“ NPD vor 1996 und der „neuen“ NPD nach 1996 zum Ausdruck kommen und nach deren Einfluss auf die begrenzten Erfolge der Partei.

Nach der Einleitung mit Ausführungen zur Problemstellung und einem Definitionskapitel zum politischen Extremismus beschreibt der Autor zunächst die Geschichte der NPD in den früheren Phasen des Aufstiegs von 1964 bis 1972 und des Niedergangs von 1972 bis 1996. Hierzu liegen mittlerweile ausführliche politikwissenschaftliche Studien vor. Brandstetter will sie bezogen auf den Zeitraum 1996 bis 2011 fortschreiben. Dazu geht er ausführlich auf die Person von Udo Voigt ein, der als geschickter Organisator des Wandlungsprozesses eine wichtige Rolle spielte. Die Mitglieder und die Struktur stehen danach im Zentrum des Interesses, wobei man selbst zu scheinbar eher marginalen Aspekten wie den Finanzen der Partei ausführliche Informationen erhält. Auch Absplitterungen und 